\title{
EchoGéo
}

41 | 2017

Un état des lieux du Brésil en 2017

\section{L'espace du politique ou la politique de l'espace : l'éviction des quartiers populaires et les Jeux olympiques de Rio de Janeiro}

\section{Cláudio Zanotelli}

\section{(2) OpenEdition}

\section{Journals}

Electronic version

URL: https://journals.openedition.org/echogeo/15083

DOI: 10.4000/echogeo.15083

ISSN: 1963-1197

\section{Publisher}

Pôle de recherche pour l'organisation et la diffusion de l'information géographique (CNRS UMR 8586)

Electronic reference

Cláudio Zanotelli, "L'espace du politique ou la politique de l'espace : l'éviction des quartiers populaires et les Jeux olympiques de Rio de Janeiro", EchoGéo [Online], 41 | 2017, Online since 28 September 2017, connection on 31 July 2021. URL: http://journals.openedition.org/echogeo/15083 ; DOI: https:// doi.org/10.4000/echogeo.15083

This text was automatically generated on 31 July 2021.

EchoGéo est mis à disposition selon les termes de la licence Creative Commons Attribution - Pas d'Utilisation Commerciale - Pas de Modification 4.0 International (CC BY-NC-ND) 


\title{
L'espace du politique ou la politique de l'espace : l'éviction des quartiers populaires et les Jeux olympiques de Rio de Janeiro
}

\author{
Cláudio Zanotelli
}

\section{AUTHOR'S NOTE}

Texte présenté lors de la séance du séminaire du programme DALVAA - Repenser le Droit à la Ville depuis les Villes du Sud - à l'université Paris-Diderot (10 mai 2016) : évictions et résistances à Accra et Rio de Janeiro.

\section{Introduction}

1 L'article mobilise les concepts d'espace politique et de justice spatiale pour révéler les contradictions insurmontables par bien des aspects entre le droit de la ville et le droit à la ville au Brésil. Pour ce faire, il portera sur le cas emblématique du quartier de Vila Autódromo, Barra da Tijuca, à Rio de Janeiro. Dans le cadre de l'accueil des Jeux Olympiques de 2016, des travaux d'aménagement pour la mise en place d'équipements et de logements pour les athlètes ont bouleversé l'espace de l'ouest de Rio, en particulier les quartiers de Barra da Tijuca et Jacarépagua. Vila Autodromo est un quartier populaire sous-intégré enclavé à proximité du Parc olympique. Comme dans des dizaines d'autres quartiers, une partie des habitants ont été relogés pour faire place aux projets d'aménagement prévus par la mairie, le gouvernement de l'État de Rio de Janeiro et l'autorité publique olympique. Cependant, cette éviction a rencontré la résistance des habitants qui refusaient de partir. Mais, malgré leur résistance, seulement une petite partie des habitants y sont restés (une vingtaine de familles) et ils 
occupent désormais de petits logements subventionnés, en accession à la propriété, et construits par la mairie dans une partie de l'ancien quartier.

Dans les années 1970, Vila Autódromo était un ancien quartier de pêcheurs qui se trouve aux abords du lac de Jacarépagua près de l'ancien circuit automobile (local aujourd'hui du parc olympique, voir illustration 1). En 1989, d'autres familles sont venues s'y établir et en 1994 le secrétariat de l'habitation de l'état de Rio de Janeiro y a installé une soixantaine des familles. En 1997, une centaine d'autres familles ont reçu des titres de propriété du gouvernement de l'État. En 1998, les familles qui vivaient près du lac ont reçu un droit d'usage de leur terrain pour une période d'un siècle. Enfin, en janvier 2005 le conseil municipal de Rio a intégré une partie du quartier dans une zone d'intérêt social qui encadre la réalisation des opérations d'urbanisme dans les quartiers populaires.

3 En 2011, 450 familles vivaient alors dans le quartier et ont été contraintes par la mairie de quitter leur habitation, malgré leur droit d'y rester. En réponse, les habitants organisés en une association de quartier ont fait appel à deux laboratoires de recherches associés aux universités Fédérales de Rio de Janeiro (NEPLAC/ETTEN/ IPPUR/UFRJ) et à l'Université Fédérale Fluminense (NEPH/UFF) pour proposer un plan alternatif à la mairie : le Plan Populaire de Vila Autódromo (2012). Le plan comprenait deux volets, l'un destiné à l'habitation et l'autre aux infrastructures. Il prévoyait, également, le déplacement d'une partie des habitations qui se trouvaient en zone protégée dans le quartier et détaillait les coûts de l'opération d'urbanisme. Le plan a reçu le prix international Urban Age Award décerné par la Deutsche Bank et par la London School of Economics (Lobino, 2016 ; Plano Vila Autódromo, 2012).

4 Ce qui s'est passé à Vila Autodromo illustre, selon nous, la structure des rapports inégaux et iniques qui existent au Brésil par rapport au droit à la ville. Il s'agira de montrer comment le droit de la ville et les plans et projets de l'État, instrumentalisent le Droit et la Justice dans une démarche de stigmatisation et d'exclusion des plus pauvres des zones valorisées par la construction d'infrastructures et d'équipements. Les conflits urbains liés aux Jeux Olympiques sont exemplaires des formes diverses de ségrégation socio-spatiale. En effet, en fonction des travaux d'infrastructures, les habitants les plus pauvres sont le plus souvent déplacés dans la périphérie de la ville (Faulhaber et Azevedo, 2015). Ces auteurs traitent des évictions à Rio dans le contexte de la Coupe du monde de 2014 et des Jeux olympiques de 2016: à ces occasions, 67000 personnes ont été déplacées sous prétexte qu'elles habitaient des zones à risque.

5 Cet article analyse tout d'abord rapidement les avancées et les limites du droit à la ville au Brésil. Ensuite nous traiterons la question du foncier urbain et de l'accès aux logements pour les plus pauvres ainsi que celle des programmes d'infrastructures et de leurs répercussions dans l'aménagement du territoire. Enfin, nous aborderons le cas du quartier de Vila Autódromo comme un cas exemplaire des contradictions dans l'application de la loi et des rapports de force imposés par les acteurs dominants qui définissent une configuration de la ville propre - dans le double sens du mot - à l'occasion des Jeux olympiques de Rio de Janeiro. Nous nous sommes basés sur des sources secondaires : un rapport de recherche inédit, des articles, des documentaires, le Plan Populaire de la Ville Autódromo, les articles de presse et une visite du site fin 2015. 


\section{L'espace politique et la politique de l'espace au Brésil : du droit formel au droit à la ville}

6 Nous définnissons l'espace comme politique en reprenant le sens que lui a donné Henri Lefebvre : l'espace comme produit social et produit de l'histoire a toujours été politique et stratégique (Lefebvre, 1972). Comme le dit Bernard Bret (2010, p. 49), se référant à la théorie de la justice de John Rawls: "Pour la géographie, dans la mesure où elle s'occupe des différenciations spatiales (c'est un savoir), et pour l'aménagement, dans la mesure où il a pour objet de transformer le territoire en fonction d'un projet de société (c'est une politique), la Théorie de la Justice constitue un fondement théorique extrêmement fécond». Ainsi, l'auteur s'interroge sur la manière d'appliquer un principe de réparation pour les injustices sociales et spatiales; il considère des espaces plus vastes que les villes, acceptant même que le développement soit inégal si c'est pour compenser les iniquités. À ce propos Soja $(2010$, p. 69) revisite la notion du droit à la ville en évoquant le cas des revendications des mouvements sociaux à Los Angeles contre les discriminations spatiales et les géographies injustes auxquelles sont relégués les plus pauvres, en particulier dans le domaine des transports publics. Ces luttes pour l'inscription dans le droit des revendications de ceux qui souffrent de l'injustice spatiale rappellent l'importance de l'action en dehors du droit et à l'intérieur du droit pour faire avancer la justice.

7 Plus que jamais aujourd'hui, l'espace urbain dans les grandes villes brésiliennes est le résultat des disputes politiques qui définissent des territoires d'intervention à géométrie variable en fonction des intérêts fonciers, immobiliers et financiers qui s'opposent aux intérêts des couches dominées de la société.

8 Nous avançons l'hypothèse d'un nouvel ordre urbain qui se constitue de manière progressive à partir des années 1990. Ce nouvel ordre est apparemment contradictoire, il est le résultat de temporalités et spatialités différentielles. Il s'inscrit, d'une part, dans un nouvel ordre du droit urbanistique supposé représenter les avancées des luttes sociales issues des années de plomb de la dictature militaire (1964-1984) et des conquêtes sociales des années 1990-2000 et, d'autre part, il s'inscrit plus récemment dans une dispute chaque fois plus violente pour l'espace urbain central et équipé par les couches dominantes de la société. Ceci malgré, comme nous le verrons, la promotion massive de l'accès à l'habitation pour les plus pauvres sous la forme d'accession à la propriété. Cependant, ces habitats sont souvent localisés en périphérie des villes, réaffirmant, ainsi, paradoxalement, la stratégie de la politique des militaires durant les années 1966-1984 d'expansion de l'accession à la propriété d'un logement pour les populations les plus pauvres. La synthèse d'une telle dialectique se traduit par un retour relatif, mais de manière différente, aux politiques d'antan d'expulsion des plus pauvres des espaces valorisés des villes.

9 En effet, si dans les années 1960-1980, l'idéologie dominante dans les villes était celle du refoulement à la périphérie des populations sous-intégrées aux structures sociales et spatiales (Valladares, 2006), il y a eu un mouvement puissant de contreproposition à cette politique publique de l'espace équivoque. Ce mouvement est le produit direct des luttes démocratiques, pour le droit à la ville et pour une réforme urbaine qui s'inscrivent dans un espace politique qu'évoquent les droits collectifs et communs de ceux qui étaient ségrégués. Suite à ces luttes (Maricato, 2011) lors de la redémocratisation du pays, les mouvements sociaux ont réussi à imposer l'inscription de 
certains aspects relatifs au droit social de la propriété [foncière ou immobilière] urbaine dans la Constitution entrée en application en 1988. L'espace du politique s'impose alors à la politique de l'espace de la ville en droit.

Ainsi, le droit à la ville et une politique de l'espace qui valorise l'intérêt collectif sont définis, du point de vue de la lettre de la loi, comme prioritaires vis à vis de la tradition du droit brésilien où le droit de la propriété avait auparavant la priorité absolue sur l'intérêt social. En droit la fonction sociale de la propriété est inscrite comme la condition même de reconnaissance du droit à la propriété (Fernandes, 2013, p. 226). La Loi 10.257 de juillet 2001, dénommée Statut de la ville, complète et détaille le dispositif du droit de la ville et de l'élaboration des Plans Directeurs des Municipalités (Municipios) (qui sont l'équivalent du Plan Local d'Urbanisme). Cette loi a instauré la séparation entre le droit de bâtir et le droit de propriété, contraignant ainsi légalement les propriétaires à se soumettre aux interêts collectifs (Fernandes, 2013, p. 226).

11 Cependant, le Statut de la ville est, pour certains, contradictoire, puisqu'il permet des dérogations au droit en ce qui concerne l'occupation du sol et laisse aussi dans le vague une série de dispositifs. À vouloir détailler des dispositifs prévus dans la Constitution et inscrire plusieurs recommandations non obligatoires, il se voit reprocher de ne pas être efficace et d'être même illusoire (Villaça, 2005).

12 Le Statut de la ville est aussi critiqué parce qu'il ne définit pas de manière claire ce qui est la fonction sociale de la propriété (Baitz, 2011). Sa définition est confiée au Plan Directeur des Municipalités qui, malgré les avancées permises par la démocratisation (la consultation de la population - obligatoire selon la législation) - est souvent un plan trop timoré et qui, le plus souvent, n'utilise pas tous les outils (droit de préemption, capture des plus-values immobilières etc.) qui permettraient de faire face aux problèmes cruciaux des villes brésiliennes : les rentes immobilières et foncières. On peut d'ailleurs s'interroger sur la manière dont de tels dispositifs pourraient être utilisés dans le cadre d'une économie utilitariste et rentiste à tous les niveaux.

13 Les Plans Directeurs des Municipalités soulèvent divers problèmes. Ils présentent un formalisme excessif, beaucoup sont approuvés sans une réelle concertation avec la population, ils ont un langage trop juridique, sont encore des plans traditionnels (trop techniques), très peu interviennent réellement sur la structure foncière et sur le marché immobilier. De même, les outils de gestion qui ont été créés par la législation ne s'inscrivent pas dans un projet de ville clairement établi (Fernandes, 2013, p. 230). En raison du procéduralisme juridique, de la montée en puissance du pouvoir des tribunaux et du conservatisme d'une partie importante de la hiérarchie de ces derniers, l'application de lois d'urbanisme qui pourraient promouvoir un droit à la ville équitable et une justice spatiale est souvent erratique et ouvre la porte à toutes les dérives qu'instaure dans plusieurs endroits un gouvernement d'exception urbain.

Néanmoins, l'ensemble des lois qui s'inscrivent dans ce nouvel ordre urbain ont présenté des avancées formelles $\mathrm{du}$ droit de la ville et quelques avancées réelles $d u$ droit à la ville : la politique de l'environnement et du patrimoine culturel, la création de zones d'intérêt social où les interventions ont produit en plusieurs endroits de réelles améliorations dans la vie des habitants des quartiers populaires. Le processus de participation est difficile pour diverses raisons, mais bien concret. Ces milliers de plans qui ont été élaborés depuis que la loi est rentrée en application ont aussi permis de produire des informations sur les villes qui, jusqu'alors, ne disposaient que de très peu de données. Une évaluation critique de l'implantation des Plans Directeurs Municipaux 
10 ans après l'entrée en vigueur du Statut de la ville, affirme qu'" une partie des plans directeurs ne sont pas effectivement le résultat d'un pacte social pour la gestion territoriale de la commune et n'instaurent pas un dialogue avec les aspects sociaux prescrits par le Statut de la ville, particulièrement les instruments de gestion du sol urbain $»^{1}$. (Santos Junior et Montandon, 2011, p. 28)

Raquel Rolnik et Erminia Maricato ${ }^{2}$ ont eu des critiques beaucoup moins nuancées sur la façon dont les plans ont été réalisés et le droit à la ville implanté notamment après 2005 (Maricato, 2011; Rolnik, 2011). À notre avis, le principal problème est lié à l'incontrôlable propriété foncière et immobilière urbaine, ce qui conditionne le succès ou l'échec d'un plan de développement urbain, en particulier dans les villes brésiliennes qui héritent d'une injustice spatiale trop flagrante.

Il est évident que la loi et le droit ne changent pas la réalité, mais peuvent être un atout dans la politique urbaine. C'est pourquoi les rapports de force sont nécessaires, puisque le droit est un champ ouvert de dispute (la jurisprudence est le résultat de ces rapports de force) pour imposer l'application du droit de la ville comme un élément fondamental de la justice spatiale. Les difficultés d'un réel progrès du droit à la ville viennent du fait que la ville est perçue souvent comme un "objet" d'accumulation où s'investit l'excédent de capital (Harvey, 2012) et qu'il y a un abîme entre l'ordre juridique institutionel $^{3}$ et les criantes réalités urbaines et environnementales au Brésil (Fernandes, 2013).

\section{La question du foncier urbain, de l'accès aux logements, des infrastructures et des équipements urbains}

17 Au Brésil la décennie écoulée (2003 à 2013) a vu la transformation de l'ordre social et urbain en parallèle à la transformation de l'ordre du droit de l'urbanisme. Avec l'arrivée au pouvoir du gouvernement de centre-gauche du Parti des Travailleurs, une série de transformations se sont produites dans le domaine social qui ont permis l'amélioration générale du niveau de vie des plus pauvres et, en particulier, ont accéléré leur consommation (voitures et biens électroménagers) et facilité leur accès aux logements. Cependant, les inégalités persistent puisque les secteurs financiers et d'exportation de biens primaires ont enregistré des gains très importants.

Le gouvernement a mis en place des politiques d'inclusion sociale, augmentant les investissements en éducation et promouvant l'accès aux études supérieures pour les classes les plus défavorisées à travers l'instauration de quotas. Il a également augmenté la capacité de gestion de l'État avec la création de milliers d'emplois dans le secteur public.

19 À côté de cette amélioration, et de l'évolution du pouvoir d'achat du salaire minimum, le gouvernement a investi énormément en infrastructures et en habitations à partir de 2006 et jusqu'à 2014 suivant un programme de relance keynesien qui cherchait à faire face à la crise financière et économique mondiale de 2008. Le taux de chômage a été à son plus bas niveau depuis des décennies ( $4,3 \%$ en 2014), en particulier celui de la main-d'œuvre employée dans le bâtiment et les travaux publics (2,3\%) (Maricato, 2015, p. 36). 
20 Les investissements pour l'accession à la propriété d'un logement (tous secteurs confondus) sont passés de 1,8 milliard de reais en 2002, dernière année du gouvernement d'inspiration néoclassique et néolibérale de Fernando Henrique Cardoso, à 112,8 millards de reais en 2014 (environ $2 \%$ du PIB de la même année), soit 62 fois plus. Entre 2002 et 2014, 544 millards de reais ont été investis au total dans l'immobilier (soit 136 milliards d'euros !)

21 Ceci a été rendu possible par le programme du gouvernement Minha Casa, Minha Vida (« Ma maison, ma vie ») au travers de fonds publics et parapublics, de fonds de garantie d'indemnité des travailleurs et de fonds des plans d'épargne. En 2014, dans le cadre de ce programme, 1,5 million de logements ont été construits et 1,7 million d'autres étaient en construction, soit au total 3,4 millions de logements. Par ailleurs, le gouvernement a depuis annoncé un objectif de construction de 2,6 millions de logements supplémentaires, mais après la crise économique et politique de 2014-2017 les investissements ont chuté ${ }^{4}$.

22 Dans le cas de la ville de Rio de Janeiro, l'éviction des quartiers populaires qui se trouvaient dans le périmètre de la construction des équipements olympiques a été suivie par le déplacement d'une partie de ses habitants vers les logements du programme Minha Casa, Minha Vida qui se trouvent majoritairement dans la région de l'extrême ouest de la ville délaissée par les infrastructures (voir à ce propos Faulhaber et Azevedo, 2015 ; Rolnik, 2015).

23 Ces programmes de logements ont provoqué un boom dans le secteur immobilier, en particulier quand ils sont associés aux grands événements sportifs (Coupe du monde de 2014 et Jeux olympiques de 2016). La relance du secteur immobilier et de l'investissement en infrastructures et des équipements publics, en particulier dans le transport urbain abandonné pendant des décennies, a entraîné dans les très grandes villes « un intense processus de spéculation foncière et immobilière » (Maricato, 2015, p. 38). Ainsi, entre janvier 2008 et janvier 2015 le prix sur le marché de l'immobilier a augmenté de $65 \%$ à Rio et de $218 \%$ à São Paulo (Maricato, 2015, p. 39).

24 Cette spéculation est en grande partie due à la non intervention des municipalités dans le marché foncier et immobilier, la législation n'étant pas appliquée ou étant contournée avec bien souvent la complicité des élus locaux. Il y a eu ainsi «une symbiose entre les gouvernements, les parlements et les capitaux immobiliers, financiers et de la construction qui ont pris d'assaut les villes » (Maricato, 2015, p. 39).

Le foncier urbain et périurbain ainsi que la spéculation autour du prix dans le secteur immobilier incontrôlé peuvent être constatés par les énormes espaces vides à l'intérieur des périmètres définis comme urbains, mais aussi dans les zones urbaines denses. Ce sont souvent des terres laissées en jachère dans l'attente d'une valorisation.

Outre les programmes d'expansion de l'immobilier, le gouvernement a lancé dès 2007 un programme keynesien d'investissement en infrastructures qui au niveau urbain a tout d'abord été destiné aux quartiers populaires visant à "l'urbanisation des favelas » pour le développement urbain (Maricato, 2011), favorisant ainsi l'accessibilité des quartiers les plus pauvres des favelas à Rio. Plusieurs étapes de ce programme ont été lancées depuis 2007 et jusqu'en 2015, en vue de soutenir l'investissement en infrastructures, mais aussi les logements sociaux. À Rio les PACs ont soutenu, parmi d'autres, les travaux et équipements de transports publics, comme une des conditions pour la réalisation des Jeux olympiques. Ainsi l'ouest de la ville, en particulier Barra da 
Tijuca qui a reçu les principaux équipements sportifs (Parc olympique) et la «vila olimpica » où logeaient les athlètes, a été connecté à la ville par l'extension d'une ligne de métro, par des lignes d'autobus en site propre (Bus Rapid Transport, BRT) et par des travaux d'aménagement urbain. Malgré une réelle amélioration du transport, ces travaux ont aussi valorisé les quartiers résidentiels huppés et ont contribué à l'expulsion des populations vivant dans des quartiers populaires situés aux abords des équipements ou des infrastructures ${ }^{5}$.

\section{Quartier de Vila Autódromo, un espace en dispute par les acteurs sociaux dans le cadre des Jeux olympiques}

La ville de Rio de Janeiro a une superfície de $1200 \mathrm{~km}^{2}$ et une population de 6,4 millions d'habitants (IBGE, 2015), $63 \%$ de ce territoire étant quasiment inoccupé (Tardin, 2008, p. 60). Les districts de Barra da Tijuca et Jacarépagua situés dans la zone ouest de Rio, comptaient en $2010,14,4 \%$ de la population de la ville et occupaient $24 \%$ de sa superficie. En 2000, $65 \%$ de la superficie de Barra da Tijuca et Jacarépagua étaient considérés comme "espaces libres » dans le sens de Tardin (2008). L'auteur considère comme espaces libres, les espaces ruraux, les forêts et les espaces urbains non occupés qu'ils soient publics ou privés (Tardin, 2008, p. 45). Le territoire est traversé de plaines côtières inondables, de grands lacs (Lacs de Tijuca e de Jacarépaguá) et de massifs montagneux (Pedra Branca et Tijuca) qui composent un paysage unique et beau.

Cette zone ouest de la ville de Rio présente un trait marquant : la ségrégation entre les populations les plus pauvres et les plus riches. Barra da Tijuca a été planifié par Lucio Costa, urbaniste de Brasilia, et garde certains des traits de la capitale du Brésil : larges avenues bordées de très hauts immeubles, centres commerciaux et résidences sécurisées (Gated cities). Les plus pauvres habitent soit dans les quartiers populaires situés à quelques kilomètres de Barra (Jacarépagua, Cidade Deus ou d'autres plus éloignés), soit en plus petit nombre dans les interstices, entre les canaux, lacs et les axes routiers ou sur les versants des mornes.

Vila Autódromo (illustration 1) se situe entre le lac de Jacarépagua et les voies rapides et le parc olympique où ont été construits plusieurs équipements pour les Jeux olympiques. Une partie de ces habitants est venue s'installer dans la Vila Autódromo lors de la construction de la Barra da Tijuca dans les années 1980, tandis qu'une autre partie, en provenance d'autres quartiers, a été installée à Vila Autódromo par les services de la mairie de Rio. Au fil du temps un certain nombre de ménages ont reçu de la part de la municipalité et du gouvernement de l'État des titres de propriété ou de reconnaissance du droit d'usage des terrains (Plano Popular, 2012). Les ménages étaient composés en grande majorité de catégories sociales dominées, mais aussi de couches moyennes. L'habitat présentait une certaine diversité en fonction des revenus des familles. 
Illustration 1 - Localisation de la Vila Autódromo à Rio de Janeiro

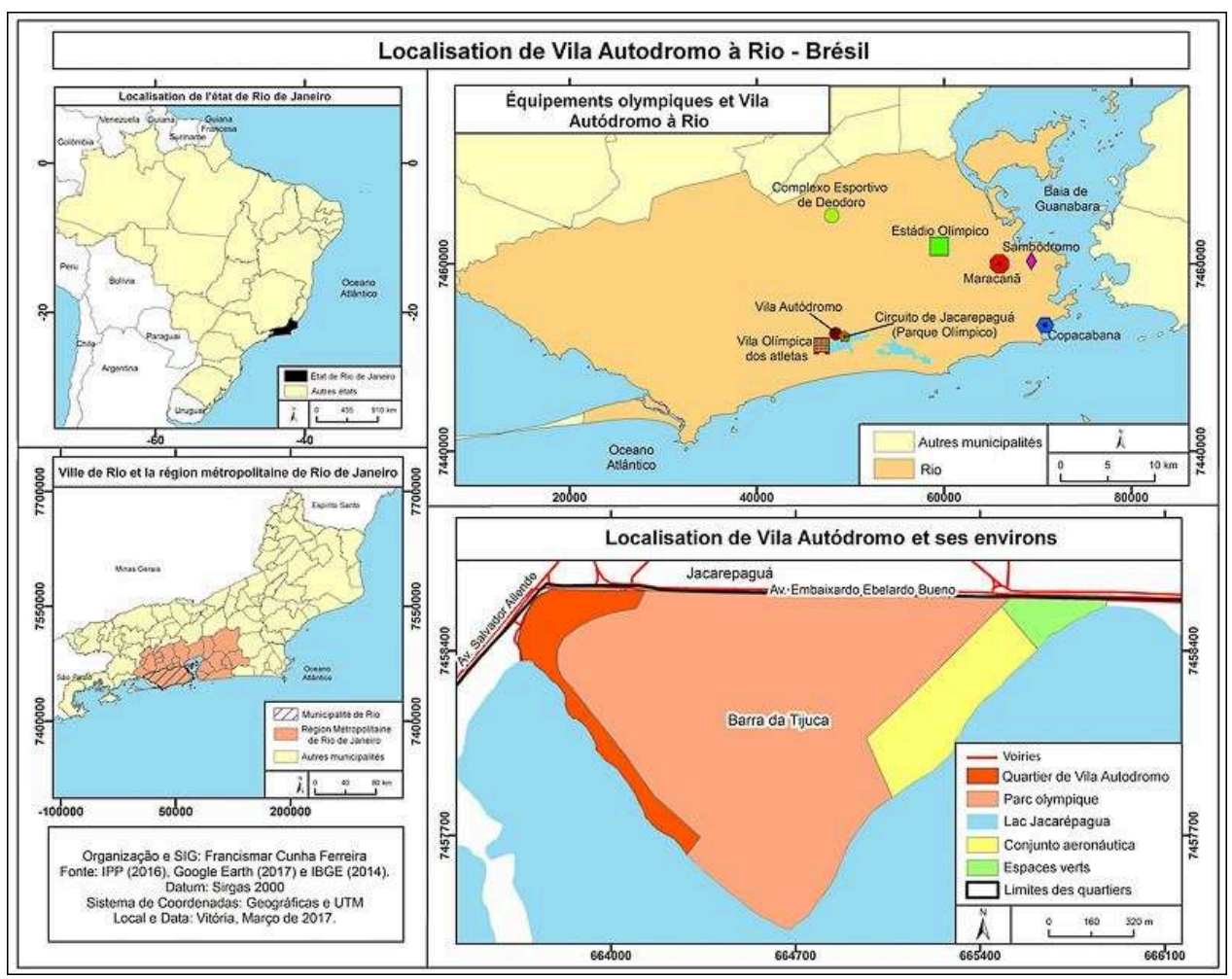

Vila Autódromo est un exemple paradigmatique de la valorisation des terrains de certains quartiers populaires en raison de la construction des grands équipements et d'infrastructures, plus particulièrement des équipements construits pour les Jeux olympiques de 2016. En effet, comme nous l'avons déjà noté, les prix se sont envolés dans la ville, conséquence des stratégies des promoteurs immobiliers et propriétaires fonciers qui ont su s'approprier les plus-values entraînées par les investissements publics et privés. Ainsi, pour la seule ville de Rio entre 2010 et 2013, 3099 ménages avaient été déplacés de leur habitat et 7834 autres étaient menacés de déplacement pour des raisons diverses (FASE, 2014, p. 20)6. D'autres auteurs ont estimé plus récemment le nombre d'habitants déplacés à 67000 habitants (Faulhabert et Azevedo, 2015).

31 Depuis les années 1990, les habitants du quartier de Vila Autódromo ont été régulièrement menacés d'éviction par les différentes majorités municipales. Rio étant choisi pour accueillir les Jeux olympiques, la municipalité a annoncé qu'elle devrait déplacer les habitants du quartier en vue de l'extension des voies rapides et des avenues qui bordent la zone (Plano Popular, 2012, p. 8). L'objectif de la mairie était de réduire de 3,5\% la superficie des favelas dans la ville de Rio et Vila Autódromo se trouvait parmi les 119 favelas qu'elle souhaitait évincer (Plano Popular, 2012, p. 8).

Les habitants du quartier se sont mobilisés pour défendre leur droit, mais la démolition de la grande majorité des maisons a eu lieu à l'approche des Jeux. Il ne restait plus que 3 habitations parmi les décombres au début de l'année 2016 (Lobino, 2016). La vingtaine de familles qui restaient ont été relogées sur place dans des maisons construites par la mairie au moment de l'ouverture des Jeux (El Pais, 26/07/2016).

33 Cette opération a été réalisée dans le non-respect du Statut de la Ville, puisque Vila Autdromo est reconnue par la mairie depuis 2005 comme Zone d'Intérêt Social. Selon la 
législation, les terrains publics doivent être, en cas de litige, prioritairement destinés aux habitants de ces zones. Ainsi, on aurait dû trouver une solution qui réponde aux souhaits des habitants. Bien sûr, des interventions étaient nécessaires, par exemple, en reconstruisant ailleurs dans le quartier les maisons localisées initialement dans une zone à risque. D'autre part, le principe selon lequel on ne déplace pas les habitants vers des zones lointaines et dépourvues de services et équipements, inscrit dans le Statut de la Ville, aurait dû être respecté.

Illustration 2 - Habitations parmi les décombres à Vila Autódromo à la fin 2014

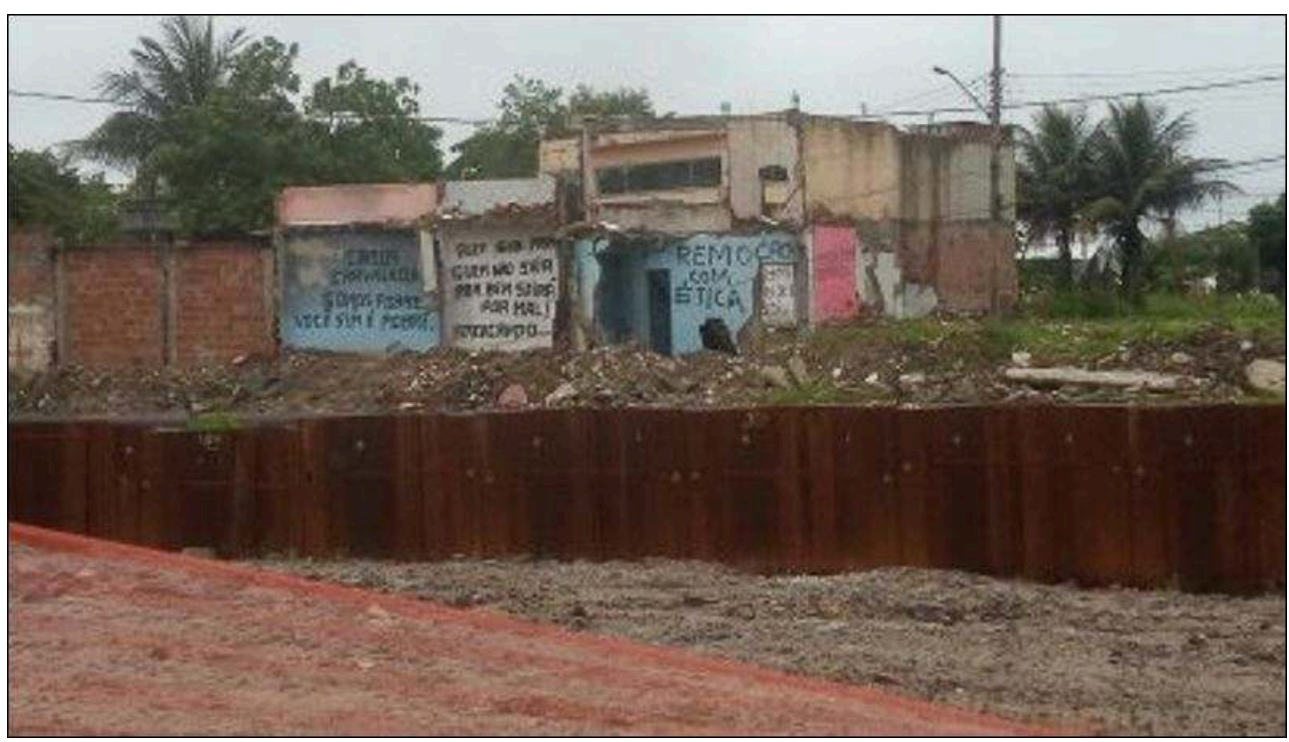

Auteur : C. Zanotelli.

En 2011, afin de trouver une solution aux propositions de démolition de Vila Autódromo, le Plan Populaire d'aménagement de Vila Autodromo qui respectait la législation et en même temps desenclavait le quartier a été élaboré. Ce plan n'a jamais été pris en compte par la mairie (Plano Popular Vila Autodrómo, 2012; Lobino, 2016).

Utilisant une stratégie déjà employée ailleurs dans la ville, notamment dans le projet autour du port de Rio (FASE, 2014), le maire a fait plusieurs annonces selon lesquelles il prétendait négocier le départ des habitants. À plusieurs reprises, il a donné l'impression de reculer, mais, finalement, il a imposé ses points de vue en utilisant des expédients qui posent question, en particulier celui de négocier avec des groupes différents d'habitants.

Après cela, la mairie a réussi à déplacer la plupart des habitants vers un quartier d'habitat populaire de Minha Casa, Minha Vida (Vila Carioca) qui se trouve à quelques kilomètres du site. D'autres habitants de Vila Autódromo, en général avec un capital économique et social plus important, qui avaient refusé de négocier leur départ, ont cédé et, pour certains, ont reçu des indemnités de plusieurs centaines de milliers de reais. Mais, une petite partie des habitants, malgré des promesses d'indemnités millionnaires en échange de leur départ du quartier, ont refusé de partir. Comme a déclaré une des dernières habitantes "nem todo mundo tem um preço " (tout le monde n'a pas un prix), en se référant au fait qu'une partie des habitants ont fini par cédé aux pressions et ont accepté des indemnisations alléchantes (Lobino, 2016, p. 10). 
La résistance des derniers habitants a été soutenue par une campagne d'innombrables personnalités et des artistes en soutien à la cause de Vila Autodromo (Entretien Lobino, avril 2016). C'est pourquoi le maire a annoncé en mars 2016 qu'il allait urbaniser ce qui restait du quartier et un protocole d'accord a été signé avec les derniers habitants. Ceci a bien été fait parce que les Jeux approchaient et que la mairie souhaitait nettoyer les gravats des démolitions qu'elle avait laissé sur place pour contraindre les habitants à partir (Lobino, 2016). Ces gravats ont été finalement retirés des rues de la Ville Autodromo et les maisons construites (Cf. illustration 4). En effet, le quartier de Vila Autódromo faisait désordre à côté du paysage idyllique du Parc olympique et des 31 tours du quartier résidentiel des athlètes (illustration 3)

Illustration 3 - Le quartier résidentiel des athlètes

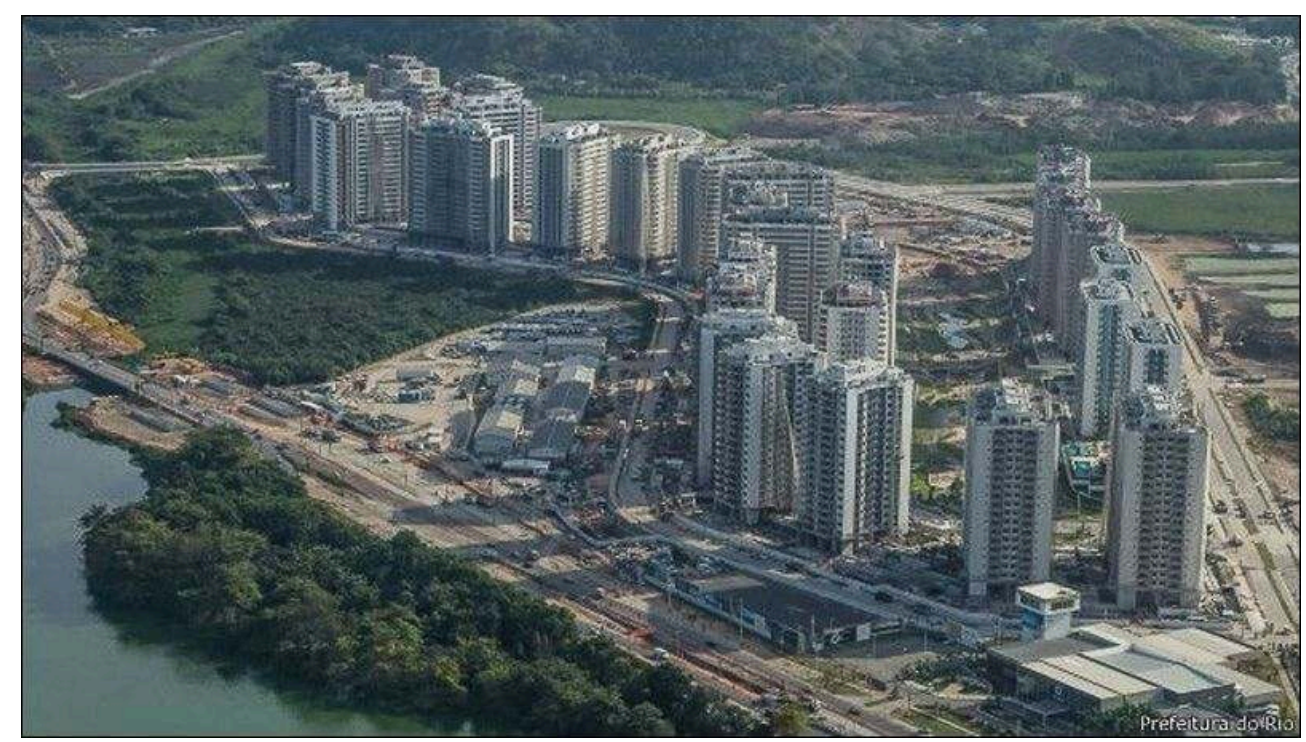

Source : http://www.brasil2016.gov.br/ptbr/megaeventos/olimpiadas/instalacoes/vila-olimpica 
Illustration 4 - Vila Autódromo et ses environs en août 2009 et juin 2016

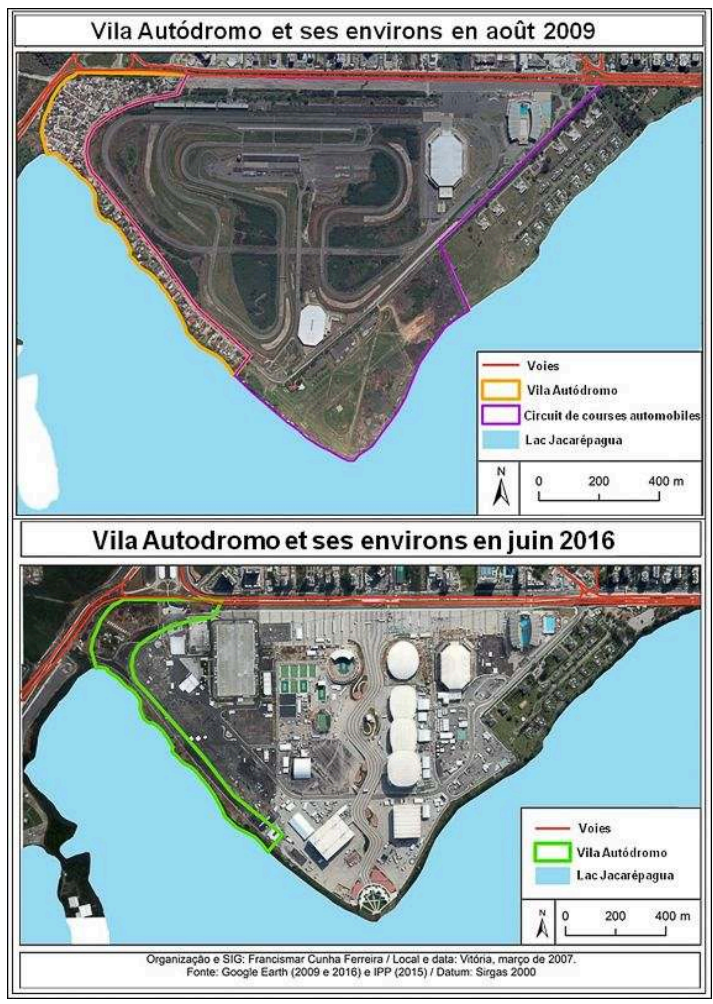

La violence concrète et symbolique envers les habitants qui résistaient à Vila Autódromo a été documentée ${ }^{7}$ et a fait l'objet de divers articles et des reportages dans la presse ${ }^{8}$.

Ce qui s'est joué dans la lutte autour de la Vila Autódromo ce sont des intérêts économiques de premier plan révélateurs de la conception que la mairie et plus largement l'État, les grandes entreprises de travaux publics et les grands propriétaires fonciers, ont de la ville et de l'urbanisme.

Le Parc olympique ainsi que le quartier de logements des athlètes (Vila olimpica) ont fait l'objet de contrats de partenariat public et privé. Deux entreprises de bâtiments et travaux publics et un gros propriétaire foncier ont signé un accord avec la mairie et l'autorité olympique pour construire une partie des installations du Parc olympique qui laisseront en partie, après les Jeux, la place à des projets immobiliers. Par ailleurs, les travaux d'infrastructures et en particulier les transports publics financés par l'État desservent le Parc et le quartier des logements des athlètes et contribuent, évidemment, à la valorisation des immeubles.

41 En échange d'un investissement estimé à 1 milliard de reais dans la construction des équipements pour les Jeux, ces entreprises pourront construire en face du quartier de Vila Autódromo des tours d'habitations et des villas de haut standing (Entretien de Mr. Carlos Carvalho à BBC Brésil, 10 aout 2015).

Mr. Carlos Carvalho est un gros propriétaire foncier et entrepreneur du BTP qui possède 1000 hectares de terres à Barra da Tijuca dont les terrains où ont été construites les 31 tours pour loger les athlètes. Il sera responsable de la mise sur le marché des 3600 appartements qui resteront vides après la fin des Jeux. (Entretien de M. Carvalho à BBC Brésil, op.cit.). Mr. Carvalho est aussi associé aux projets pour le Parc olympique. Lors d'un entretien avec les journalistes il a avoué être d'accord avec la 
mairie : "On ne peut pas habiter dans un appartement et vivre avec un indien à côté, par exemple. Nous n'avons rien contre les indiens, mais il y a certaines choses qui ne sont pas possible. Si les gens sentent mauvais je ne resterai pas à leur côté.» (BBC, op. cit.).

En réponse à cet entretien, un habitant de Vila Autodromo a déclaré qu'ils devraient promouvoir une action en justice contre ce monsieur puisque « nous servons pour eux, mais pour habiter tout près nous sommes sales et sentons mauvais » (Cité par Lobino, 2016).

Dévoilant ses intentions Mr. Carvalho a déclaré dans un entretien au The Gardien qui a provoqué des remous, que Barra da Tijuca représente le «nouveau Rio de Janeiro, ville d'élite, du bon goût et que pour cette raison l'« île Pure " [le projet immobilier où se trouve la ville des athlètes] doit être un habitat noble et non destiné à une habitation pour les plus pauvres ».

\section{Conclusion}

Nous avons constaté que le droit est appliqué de manière partielle dans le cas de Vila Autodromo et que face au droit à la ville se trouve un droit de la ville, instrumentalisé par la mairie et les agents dominants, qui favorise la rente fonciere et immobiliere au détriment des couches dominées. L'État à tous les niveaux ne déploie que très rarement les outils de la législation afin de contrôler les spéculations et permet que des terrains publics soient la proie du secteur immobilier, comme c'est le cas de Vila Autodrómo. La politique d'éviction des pauvres du cœur des villes et des zones bien équipées et bien desservies qui avait été interrompue dans le passé semble reprendre le dessus à la faveur de la croissance des investissements et des politiques publiques qui se détournent des intérêts collectifs, malgré l'existence d'une législation progressiste et des pratiques sociales de résistance.

Il semble que le cycle des luttes populaires connaisse un net recul après les acquis sociaux accordés par le Parti des Travailleurs pendant 13 ans de gouvernement. Une certaine démobilisation favorisée par l'idéologie consumériste envahissante et par la cooptation des cadres et des leaders des mouvements sociaux par l'État, a permis que les couches dominantes imposent leurs vues même dans le cadre d'un gouvernement de centre-gauche au niveau fédéral.

Des reflexes anti-populaires des classes dominantes et d'une partie des couches moyennes sur un fond de tradition patrimonialiste et clientéliste réaffirment le pouvoir sur les hommes et la ville: le contrôle et la jouissance de la propriété foncière et immobilière.

Dans ces conditions l'espace politique est le pur produit des rapports de force qui font fi de la législation et, de cette manière, impose les interêts spéculatifs face au bien commun.

Il semble que le cycle d'une nouvelle expansion de capitaux investis dans la recherche de la rente urbaine réaffirme un nouveau cycle d'éviction des plus pauvres des zones occupées depuis des décennies au travers de l'accumulation par dépossession. Ce qui a été écrit par Lefebvre apparait, ainsi, comme irréalisable : le droit à la ville « ne peut se formuler que comme droit à la vie urbaine, transformée, renouvelée. Que le tissu urbain enserre la campagne et ce qui survit de vie paysanne, peu importe, pourvu que 
"l'urbain », lieu de rencontre, priorité de la valeur d'usage, inscription dans l'espace d'un temps promu au rang de bien suprême parmi les biens, trouve sa base morphologique, sa réalisation pratico-sensible » (Lefebvre, 1972, p. 121).

L'économie politique de l'espace promue par la Constitution et par le Statut de la Ville peuvent représenter un progrès dans le droit à la ville, néanmoins, comme écrit David Harvey "entre droits égaux qui décide est la force» (Harvey, 2012, p. 31). En effet, même si nous supposons que les politiques de soutien aux plus pauvres pourraient favoriser leur intégration dans la ville et, en partie, favoriser, par exemple, la construction des logements salubres comme le programme Ma Maison, Ma Vie, les structures d'accès aux services et aux équipements et les infrastructures des villes, majoritairement, au Brésil, continuent d'être définies, en dernière instance, par le rapport de force associé au contrôle du foncier urbain et à la valorisation immobilière sans entraves significatives. L'idéologie même, dominante et envahissante, de l'accession à la propriété est structurelle et stimulée depuis les années 1960. Comme disait un ancien ministre du régime militaire : « pour que les gens n'aient pas à se mêler des mouvements de contestation sociaux on leur donnera la possession d'une maison ", idée venue des États Unis dès le début des années 1920 (Harvey, 2012) et qui recouvre quelque chose de structurel, la ville vue comme un lieu d'accumulation du capital.

51 Ceci nous amène à nous interroger : en invoquant de manière fréquente les droits, ne serions-nous pas en train de légitimer un ordre juridique qui, d'une façon ou d'une autre, favorise en dernière instance les couches dominantes?

Ainsi, dans le cadre des rapports ancestraux de domination au Brésil, lorsque nous invoquons un «droit de/à la ville ", de droits établis dans la législation ou lorsque nous souhaiterions qu'ils puissent y être inscrits, ne serions-nous pas en train de nous lier à un ordre juridique qui de fait - même s'il y a quelques progrès ici ou là - fini grosso modo par valider l'ordre dominant? Ceci ne nous amenerait pas à croire en un « contrat social » en trompe l'oeil cristalisé dans la Constitution? Ainsi ne serions-nous pas liés à une croyance dans l'ordre, croyance que nous pensons être la nôtre, quand de fait, elle est contrôlée par les instances de l'État au service des catégories dominantes de la société ? Ou devrions-nous garder espoir et imaginer un autre droit pour une autre ville qui soit le produit des luttes sociales, dans la tradition de ce qu'on a appelle au Brésil les mouvements sociaux urbains et la reforme urbaine du passé ?!

\section{BIBLIOGRAPHY}

Bret B., Gervais-Lambony Ph., Hancock C., Landy F. (sous la direction), 2010. Justice et injustices spatiales. Paris, Presses universitaires de Paris Ouest.

Bret B., 2010. L'universalisme rawsien confronté à la diversité du réel. In Bret B, Gervais-Lambony PH, Hancock C., Landy F. (sous la direction), Justice et injustices spatiales, Paris, Presses universitaires de Paris Ouest, p. 29-54. 
Documentaire Profissão Reporter, TV Globo, 2015 consultable en ligne sur http://g1.globo.com/ profissao-reporter/noticia/2015/08/obras-polemicas-marcam-contagem-regressiva-paraolimpiadas-do-rio.html. Accès le 1/3/2017.

El Pais, 26/07/2016. Vila Autódromo, a comunidade que venceu os Jogos Olímpicos, consultable en ligne sur http://brasil.elpais.com/brasil/2016/07/25/politica/1469450857_996933.html, accès le $1 / 3 / 2017$.

Entretien de Mr. Carlos Carvalho à BBC Brésil, 10 aout 2015. Consultable en ligne sur : http:// www.bbc.com/portuguese/noticias/2015/08/150809_construtora_olimpiada_jp, accès le 22 avril de 2016.

Faulhauber L., Azevedo L. SMH, 2015. 2016: Remoções no Rio de Janeiro Olímpico. 1 ed., Rio de Janeiro, Mórula.

Fernandes E., 2013. Estatuto da cidade. Mais de dez anos depois, Ver, Universidade Minas Gerais, vol. 20, $\mathrm{n}^{\circ} 1$, p. 212-213, jan/jun.

Harvey D., 2012. Rebel cities: from the right to the city to the urban revolution. Londres, Verso.

Lefebvre H., 1972. Le droit à la ville. Paris, Anthropos.

Lobino C, 2016. Entre as margens do lago e a margem do Estado: a mediação pela violência no caso de Vila Autódromo - Rio de Janeiro. Texte inédit.

Maricato E, 2011. O impasse da política urbana no Brasil. São Paulo, Editora Vozes, Editora Expressão Popular.

Maricato E., 2015. Para entender a crise urbana. São Paulo, Editora Expressão Popular.

Ministério do Planejamento, PAC, consultable en ligne sur http://www.pac.gov.br/obra/78165, accès le 22/08/2017.

Obras de mobilidade para olimpíada recebem R\$7,1 bilhões do governo federal, Rio 2016, Portal Brasil, consultable en ligne sur http://www.brasil.gov.br/infraestrutura/2016/03/mobilidadepara-olimpiada-no-rio-recebe-r-7-1-bilhoes-do-governo-federal , accès le 21/08/2017.

Plano Popular Da Vila Autodromo, Associação dos Moradores e pescadores da Vila Autódromo, 2013.

Ribeiro R., 2014. Avaliação da execução do programa de aceleração do crescimento (PAC) (2007/2014), Nota técnica 15/2014. Câmara dos deputados, consultable en ligne sur http://www2.camara.leg.br/ orcamento-da-uniao/estudos/2014/nt15.pdf, accès le 22/08/2017.

Rolnik R., 2015. Guerra dos lugares, A colonização da terra e da moradia na era das finanças. São Paulo, Boitempo.

Santos Junior O. A., Montandon D. T. 2011. Os Planos Diretores Municipais Pós-Estatuto da Cidade: balanço crítico e perspectivas. Rio de Janeiro, Observatório das Metrópoles/Letra Capital.

Soja E., 2010. La ville et justice spatiale. In Bret B, Gervais-Lambony PH, Hancock C., Landy F (Sous la direction), Justice et injustices spatiales. Paris, Presses universitaires de Paris Ouest, p. 55-74.

Tardin R., 2008. Espaços livres: sistema e projeto territorial. Rio de Janeiro, 7 Letras.

Vainer E, Harvey D, Maricato E. et al., 2013. Cidades rebeldes. São Paulo, Editora Boitempo.

Vainer C., Maricato E. et al., 2014. Brasil em jogo. São Paulo, Carta Capital.

Valladares L., 2006. La favela d'un siècle à l'autre. Ed. Sciences de l'homme, Paris. 
Vasconcelos L.F., Gonçalves I. Assim funciona a contra-reforma urbana, GGN, O jornal de todos os brasis, consultable en ligne sur http://jornalggn.com.br/noticia/assim-funciona-a-contra-reformaurbana-por-luiz-fernando-vasconcelos-e-isabella-goncalves , accès le 21/08/2017.

Vídeo Justice Global, consultable en ligne sur http://puc-riodigital.com.puc-rio.br/Televisao/ Cidade/Comunidade-da-Vila-Autodromo-vive-o-drama-da-remocao-19241.html\#.U6nNWfldWYE, accès le $1 / 3 / 2017$.

\section{NOTES}

1. Ce rapport a mis aussi en exergue, parmi d'autres problèmes, l'« inadéquation des outils prévus dans les plans en ce qui concerne (...) l'induction du développement urbain.» (Santos Junior Et Montandon, 2011. p. 31-34).

2. Universitaires issues de la mouvance de la réforme urbaine et qui, entre 2003 et 2005, ont eu des responsabilités au plus haut niveau du premier gouvernement Lula, de 2003 à 2005, au Ministère de la Ville, la première a été secrétaire exécutive du ministère et la deuxième secrétaire nationale des programmes urbains.

3. L'ordre institutionnel est en pleine mutation et en pleine déconfiture actuellement au Brésil, comme le démontre le décret no.759 du gouvernement (Medida Provisória) daté du 22 de décembre 2016 qui traite de la régularisation foncière et prétend promouvoir l'expansion du crédit aux consommateurs auprès des banques à travers la mise sur le marché des propriétés immobilières faisant l'objet d'une régularisation dans les quartiers sous-intégrés, mesure qui peut provoquer une accentuation de la ségrégation socio-spatiale des populations plus pauvres les poussant à vendre leur logement et aller habiter ailleurs. Voir à ce propos l'article de Luiz Fernando Vasconcelos e de Isabella Gonçalves, Assim funciona a reforma urbana, GGN, O Jornal de todos os brasis, 15/05/2017.

4. Près de $47 \%$ de ces habitations étaient destinées aux ménages qui ont des revenus plafonnés à 1600 Reais mensuels, soit, environ 400 Euros au taux de change du mois d'avril 2016. Ces habitations sont subventionnées à hauteur de $90 \%$ et ont une surface comprise entre $37 \mathrm{~m}^{2}$ et $43 \mathrm{~m}^{2}$. Leur prix de revient est défini préalablement par le gouvernement et les entrepreneurs doivent se soumettre à un cahier de charge strict. La limitation des coûts des logements entraînent la recherche de terrains disponibles dans les franges de l'agglomération, en général sans infrastructures et équipements. Ces terrains sont soit cédés gratuitement par les municipalités, soit achetés à très bas prix par les entreprises du bâtiment. Le reste du programme (53\%) est aussi subventionné et a un taux d'intérêt réduit ; il est destiné aux familles qui ont un revenu compris entre 1600 et 5000 reais et se situe dans des endroits plus centraux. Des modifications introduites par le gouvernement de Michel Temer depuis son accession au pouvoir en 2016 ont augmenté le plafond de revenus et modifié la valeur des immeubles pouvant être financés; ce programme d'habitations est délaissé.

5. Dans tout le pays pour la période de 2007 et 2014 ont été prévus des investissements publics, parapublics et privés de l'ordre de 1,5 mille millards de reais (405 milliards d'Euros au taux de 3,7 reais l'Euro en aout 2017) qui ont seulement en partie été suivis d'effets. L'État de Rio de Janeiro a été un de ceux qui a reçu le plus d'investissements, près de $6 \%$ du total effectivement réalisé par l'État brésilien (Cf. Ribeiro R., Note technique 15/2014 de la Chambre des députes, 2014). Pour les investissements à Rio on peut consulter la page du Ministère de la planification, PAC, 2017. Pour leses travaux d'extension de la ligne de métro vers Barra da Tijuca et la construction du Tramway du centre-ville de Rio, 7,1 millards de Reais sur un total de 10,3 milliards ont été investis par le gouvernement fédéral (Cf. Portal Brasil, 07/03/2016). 
6. Sur la débauche d'argent, l'éviction des populations pauvres, la valorisation du marché immobilier et du foncier et le non respect aux différentes législations lors des grands évenéments sportifs voir la publication Brasil em jogo (2014).

7. Documentaire Profissão Reporter, TV Globo, 2015.

8. Pour un aperçu voir Lobino, 2016.

\section{ABSTRACTS}

L'article mobilise le concept d'espace politique pour révéler les contradictions insurmontables par bien des aspects entre le «droit de la ville » et le «droit à la ville » au Brésil. Pour ce faire, il portera sur le cas emblématique du quartier de Vila Autódromo, à Rio de Janeiro. Dans le cadre de l'accueil des Jeux Olympiques de 2016 à Rio, des travaux d'aménagement pour la mise en place du Parc olympique et du quartier des logements des athlètes et de ses environs ont bouleversé l'espace de l'ouest de Rio, en particulier les quartiers de Barra da Tijuca et Jacarépagua. Vila Autodromo est un quartier populaire sous-intégré et enclavé à proximité du Parc olympique et a été en bonne partie démoli par la ville avant les Jeux. Ce qui s'est passé à Vila Autodromo illustre, selon nous, la structure des rapports inégaux et iniques qui existent au Brésil par rapport au droit à la ville. Il s'agira de montrer comment le droit de la ville, les plans et les projets de l'État, instrumentalisent le Droit et la Justice dans une démarche de stigmatisation et d'exclusion des plus pauvres des zones valorisées par des infrastructures et des équipements.

\section{INDEX}

Mots-clés: politique de l'espace, Rio de Janeiro, olympiades, éviction des quartiers populaires, justice spatiale

\section{AUTHOR}

\section{CLÁUDIO ZANOTELLI}

Cláudio Zanotelli, clzanotelli@yahoo.com.br, est Professeur titulaire du Departement et du Master et Doctorat en Géographie de l'Univérsité Féderale de l’Espírito Santo (Brésil). 